\title{
Two-dimensional carbon nanostructures obtained by laser ablation in liquid: effect of an ultrasonic field
}

\author{
L. Escobar-Alarcón ${ }^{1} \cdot$ M. E. Espinosa-Pesqueira ${ }^{2}$ • D. A. Solis-Casados ${ }^{3} \cdot$ J. Gonzalo $^{4} \cdot$ J. Solis $^{4} \cdot$ M. Martinez-Orts $^{4}$. \\ E. Haro-Poniatowski ${ }^{4,5}$
}

Received: 28 September 2017 / Accepted: 6 January 2018

(c) Springer-Verlag GmbH Germany, part of Springer Nature 2018

\begin{abstract}
The ablation of a carbon target immersed in deionized water, in absence and in presence of ultrasonic waves is reported, and the differences investigated. The obtained nanostructures are characterized by transmission electron microscopy, Raman spectroscopy and photoluminescence. Transmission electron images reveal that the produced carbon nanostructures, with and without ultrasonic excitation, are graphene-like sheets with improved quality in the first case. Samples prepared with ultrasounds show graphene layers with large sizes (several microns) and regular shapes, whereas the samples prepared without ultrasounds show smaller sizes and irregular shapes; additionally, some dispersed quasi-spherical nanoparticles are observed in the samples prepared without ultrasound. Photoluminescence measurements of the obtained nanostructures reveal emission in the blue spectral region.
\end{abstract}

\section{Introduction}

Two-dimensional (2D) nanomaterials are of great interest because at nanoscale surface effects are enhanced allowing potential applications in areas such as catalysis owing to their very high specific area. This interest has prompted the research in 2D layered materials as graphene due to its high electron mobility, enormous specific surface area, very high thermal conductivity and super-strong mechanical properties [1]. Graphene is a two-dimensional monolayer of carbon

L. Escobar-Alarcón

luis.escobar@inin.gob.mx

1 Departamento de Física, Instituto Nacional de Investigaciones Nucleares, Apdo. Postal 18-1027, 11801 Mexico City, Mexico

2 Departamento de Tecnologia de Materiales, Instituto Nacional de Investigaciones Nucleares, Apdo. Postal 18-1027, 11801 Mexico City, Mexico

3 Universidad Autónoma del Estado de México, Facultad de Química, Paseo Colon esq. Paseo Tollocan S/N, 50120 Toluca, Estado de México, Mexico

4 Laser Processing Group, Instituto de Optica, IO, CSIC, Serrano 121, 28006 Madrid, Spain

5 Departamento de Física, Universidad Autónoma Metropolitana Iztapalapa, Apdo. Postal 55-534, Mexico City, Mexico atoms being the structural base of diverse carbon allotropes including graphite, carbon nanotubes and fullerenes. A wide variety of methods to synthesize graphene have been reported. These include mechanical exfoliation [2], chemical exfoliation [3], liquid exfoliation [4] as well as CVD [5] and PVD techniques [6]; however, one of the remaining issues concerns the preparation of this nanomaterial with properties such as crystallinity, shape or size distribution in a controlled way. Therefore, the investigation of alternative synthesis routes to develop high-quality and large-area graphene with controllable layer thickness is still a challenge.

Laser ablation in liquids has become an alternative route for the synthesis of nanostructures for many applications [7]. This technique has been widely used to produce a variety of nanostructures with different shapes and sizes including metals [8], alloys [9], and oxides [10], among others. An important advantage of this method is its versatility enabling its combination with other techniques in an easy way. However, in spite of its experimental simplicity, the basic mechanisms of nanostructures formation involve processes of high complexity in the fundamental understanding that still remain under investigation [7]. Carbon nanostructures in various forms and sizes have been prepared from graphite by liquid phase laser ablation [11, 12]. Graphene sheets have been synthesized using a Nd:YAG laser in liquid nitrogen and distilled water $[13,14]$. Additionally, the preparation of graphene by PLD under different conditions has been 
reported $[15,16]$. On the other side, ultrasound exfoliation of bulk-layered materials has been applied as a route for the preparation of 2D nanostructures including graphene [17, $18]$.

The combination of laser ablation in liquids with an ultrasound field has already been investigated for dispersion purposes [19], to study the effects of the ultrasonic excitation on the structure of laser-ablated nanoparticles [20] and it has been reported to lead to an enhancement of the production rate of the nanoparticles [21]. In a previous work, we have reported that ultrasound not only generates dispersion and increases the yield in the nanoparticle formation but also produces important changes in the morphology of the nanostructures producing nanosheets in colloidal suspensions [22]. In this work, it is shown that the use of liquid laser ablation assisted by an ultrasonic field allows the preparation of $2 \mathrm{D}$ carbon nanosheets formed by single and few layers of graphene.

\section{Experimental procedure}

Two-dimensional carbon nanostructures were prepared by laser ablating a high-purity (99.99\%) commercial polycrystalline graphite disk target, diameter of $2.5 \mathrm{~cm}$ and thickness of $0.6 \mathrm{~cm}$, submerged in distilled water. The target was placed inside a glass cylindrical container with an inner diameter of $2.5 \mathrm{~cm}$ to fit the target at the bottom. Afterwards, $5 \mathrm{ml}$ of distilled water were added forming a layer with a thickness close to $1 \mathrm{~cm}$ over the target surface. For the experiments with ultrasonic excitation, the container was placed inside an ultrasonic bath working at a frequency of $40 \mathrm{kHz}$ and a power of $70 \mathrm{~W}$. The laser beam was directed by means of mirrors and focused onto the target surface passing through a quartz lens with $12 \mathrm{~mm}$ of focal length. Laser ablation was carried out using the second harmonic of a $\mathrm{Nd}$ :YAG laser at a wavelength of $532 \mathrm{~nm}$ and $5 \mathrm{~ns}$ of pulse duration, working at a repetition rate of $10 \mathrm{~Hz}$. Experiments at different laser fluences, $0.5,2.2$ and $3.6 \mathrm{~J} / \mathrm{cm}^{2}$, with and without the presence of an ultrasonic field were performed. Under these experimental conditions, we are below the plasma formation threshold according to the model of Ren [23] The ablation time was kept constant at 15 min for all samples.

To characterize the obtained nanostructures by transmission electron microscopy (TEM), a drop of each of the prepared colloids was deposited on TEM 300 mesh copper grids covered with carbon film. These samples were analyzed (TEM JEOL 2100) with a point resolution of $0.19 \mathrm{~nm}$, at an operating voltage of $200 \mathrm{kV}$. The same procedure was repeated on pieces of silicon wafers for optical microscopy and Raman spectroscopy characterization. Raman spectroscopy was used to study the vibrational features of the nanostructures using a HR LabRam 800 system equipped with an Olympus BX40 confocal microscope; Raman spectra were acquired using a 100X objective lens with a $532 \mathrm{~nm}$ laser excitation; the optical images were obtained using this microscope. Optical absorption measurements were performed using a Perkin Elmer lambda-35 spectrometer. Photoluminescence properties of the carbon-based colloids were studied by PL spectroscopy using a spectrofluorometer (FluoroMax 4, Horiba Jobyn Ivon) equipped with a 150W Xenon lamp as excitation source; samples were excited using a wavelength of $320 \mathrm{~nm}$.

\section{Results}

The obtained colloids showed a characteristic coloration depending on the preparation conditions. Colloids were almost transparent in the absence of ultrasonic excitation, whereas they were light brown in the presence of the ultrasonic field. This result suggests a greater yield production of carbon nanostructures with the ultrasonic field in agreement with previous reports [21]. To guarantee that the obtained 2D-nanostructures were not produced by mechanical exfoliation of the graphite target, it was treated in the ultrasonic field for 30 min and subsequently a drop of the solution was observed by TEM. No evidence of exfoliated material was found in this case.

\subsection{Structural characterization}

Samples prepared without ultrasound assistance consisted of carbon agglomerates as well as small particles. Instead, samples produced with an ultrasonic field contain a significant concentration of 2D structures like sheets with sizes of several microns and regular shapes. This is illustrated in Fig. 1 for the case of the lowest fluence $\left(0.5 \mathrm{~J} / \mathrm{cm}^{2}\right)$. Samples prepared at higher laser fluences showed similar features.

Figure 2 shows representative TEM images of the samples produced with and without ultrasonic excitation to compare their characteristics in more detail. Sheets and particles with irregular shape forming agglomerates, as well as $2 \mathrm{D}$ nanostructures with lateral dimensions up to $2 \mu \mathrm{m}$ are observed in absence of ultrasonic excitation (Fig. 2a). Instead, when laser ablation in liquid media is performed in the presence of the ultrasonic field, samples were more dispersed and much less agglomerated and contained a significant concentration of 2D nanosheets (Fig. 2b). It is important to remark the presence of carbon single layers in this image in which some carbon sheets reach sizes close to $15 \mu \mathrm{m}$ and exhibit very regular shapes. This can be more clearly seen in Fig. 2c, which shows a 2D structure that presents these features. Additionally, this TEM image reveals the presence of regions in which 1,2,3 and even 4 graphene 


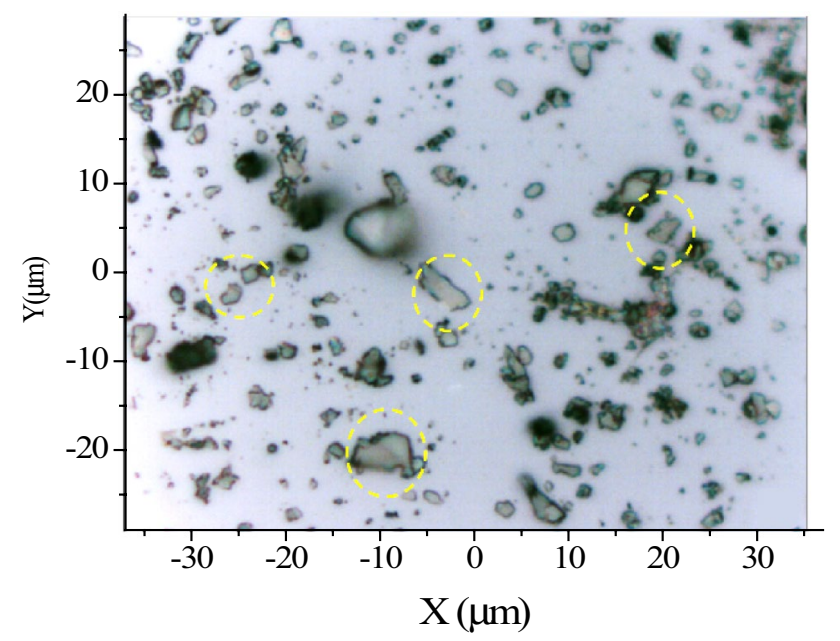

Fig. 1 Optical microscopy image of the sample obtained at a laser fluence of $0.5 \mathrm{~J} / \mathrm{cm}^{2}$ with the assistance of ultrasonic excitation. Dashed circles show some of the observed 2D structures

layers are overlapped (labeled by the corresponding number in the figure). Figure $2 d$ shows the electron diffraction pattern of a single layer (region labeled as "*”). The typical six-fold symmetry expected for graphene is observed, revealing the high degree of crystallinity of the $2 \mathrm{D}$ carbon sheet. This result indicates that high-quality graphene can be obtained using this experimental configuration. The above results suggest that the presence of the ultrasonic field during laser ablation in liquid promotes the exfoliation of graphene nanosheets having from 1 to a few layers. Finally, it is worth mentioning that nanostructures formed with more than a few layers were rarely observed in the different samples prepared under ultrasonic excitation.

We have completed the structural characterization using Raman spectroscopy, since this allows the characterization of the different forms of carbon-based materials such as graphite, diamond, carbon nanotubes, fullerenes, amorphous carbon and graphene [24-26]. In general terms, their spectra share common features in the $1000-3000 \mathrm{~cm}^{-1}$ region; the so-called D, G and 2D peaks close to 1340,1560 and $2700 \mathrm{~cm}^{-1}$, respectively. The position, peak shape and relative intensity between them are used to determine what kind of carbon structure is present [25]. Figure 3 shows the Raman spectra upon excitation at $532 \mathrm{~nm}$ of samples
Fig. 2 TEM images of the carbon nanostructures present in samples prepared at $0.5 \mathrm{~J} /$ $\mathrm{cm}^{2}$ : a without, and with $\mathbf{b}$ ultrasonic excitation; c zoom of the previous image; $\mathbf{d}$ electron diffraction pattern of a singlelayer graphene indicated by "asterisk" in c
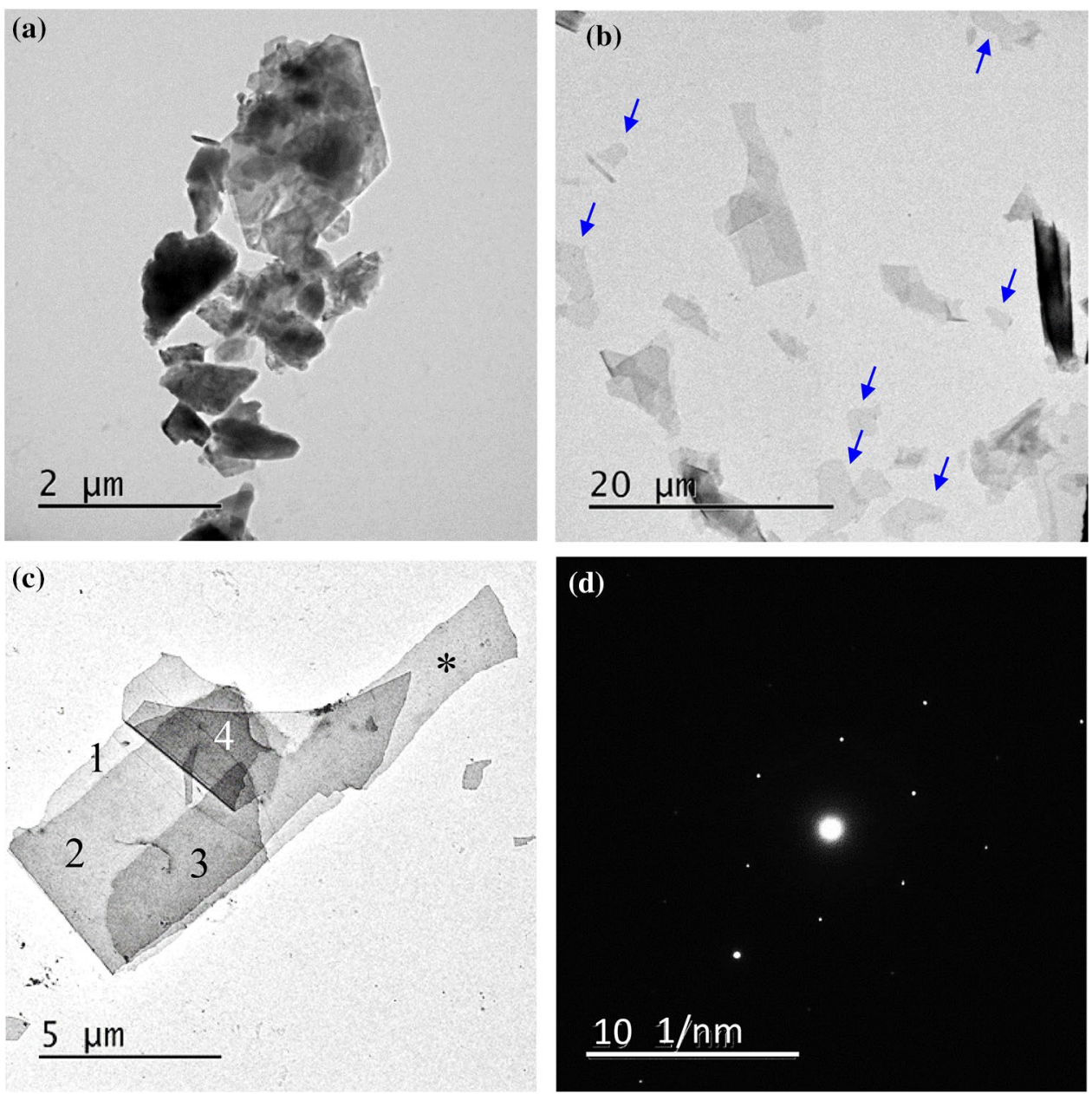


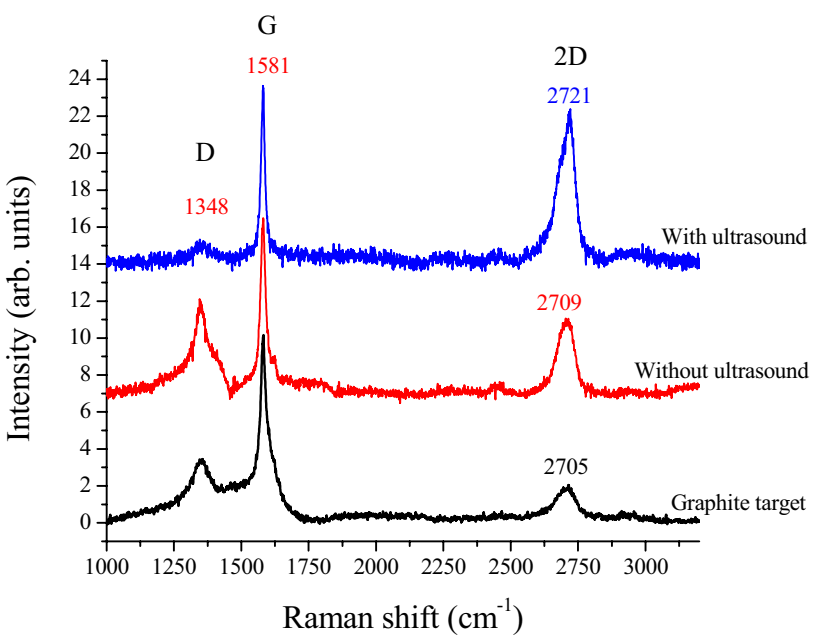

Fig. 3 Raman spectra of the carbon target and two samples produced by ablation in liquid at $0.5 \mathrm{~J} / \mathrm{cm}^{2}$ with and without ultrasonic excitation. The position of the $\mathrm{D}, \mathrm{G}$ and $2 \mathrm{D}$ bands are indicated in the figure. The intensities of the spectra have been normalized to that of the $\mathrm{G}$ peak

prepared at the same laser fluence $\left(0.5 \mathrm{~J} / \mathrm{cm}^{2}\right)$ with and without ultrasonic excitation as well as the corresponding to the polycrystalline graphite target. To reveal clearly the differences between the Raman spectra of the samples, these were scaled to have similar intensity of the $G$ peak close to $1581 \mathrm{~cm}^{-1}$. Several differences are clearly observed. First, an evident higher intensity of the $\mathrm{D}$ band in the sample prepared without ultrasound is seen. This band arises because of the presence of defects or disorder [25, 26]; thus indicating a higher degree of disorder in this sample. This is consistent with the microscopy results because samples prepared in the absence of ultrasonic excitation are formed by agglomerates of mixtures of carbon nanosheets and nanostructures with irregular shapes. The very low intensity of the $\mathrm{D}$ band for the sample obtained in the presence of the ultrasonic field is consistent with good quality $2 \mathrm{D}$ carbon nanostructures almost free of defects [25]. Second, it is seen that the 2D peak drastically increases in samples prepared by liquid laser ablation. When compared to the polycrystalline graphite target, in the sample produced at $0.5 \mathrm{~J} / \mathrm{cm}^{2}$ without ultrasonic excitation the 2D peak intensity increases by a factor of $\sim 2.5$, while for the sample with ultrasonic excitation increases by a factor of $\sim 5$. Since graphene has a sharp 2D peak roughly four times more intense than the $G$ peak [26], the fact that the $2 \mathrm{D} / \mathrm{G}$ peak intensities ratio is higher for the samples prepared in an ultrasonic field than in absence of ultrasonic excitation can be taken as a clear indication of the higher graphene content, even if graphite substantially contributes to the $2 \mathrm{D}$ band in the samples produced with ultrasonic excitation. To perform a deeper analysis of the Raman spectrum of samples prepared in an ultrasonic field,
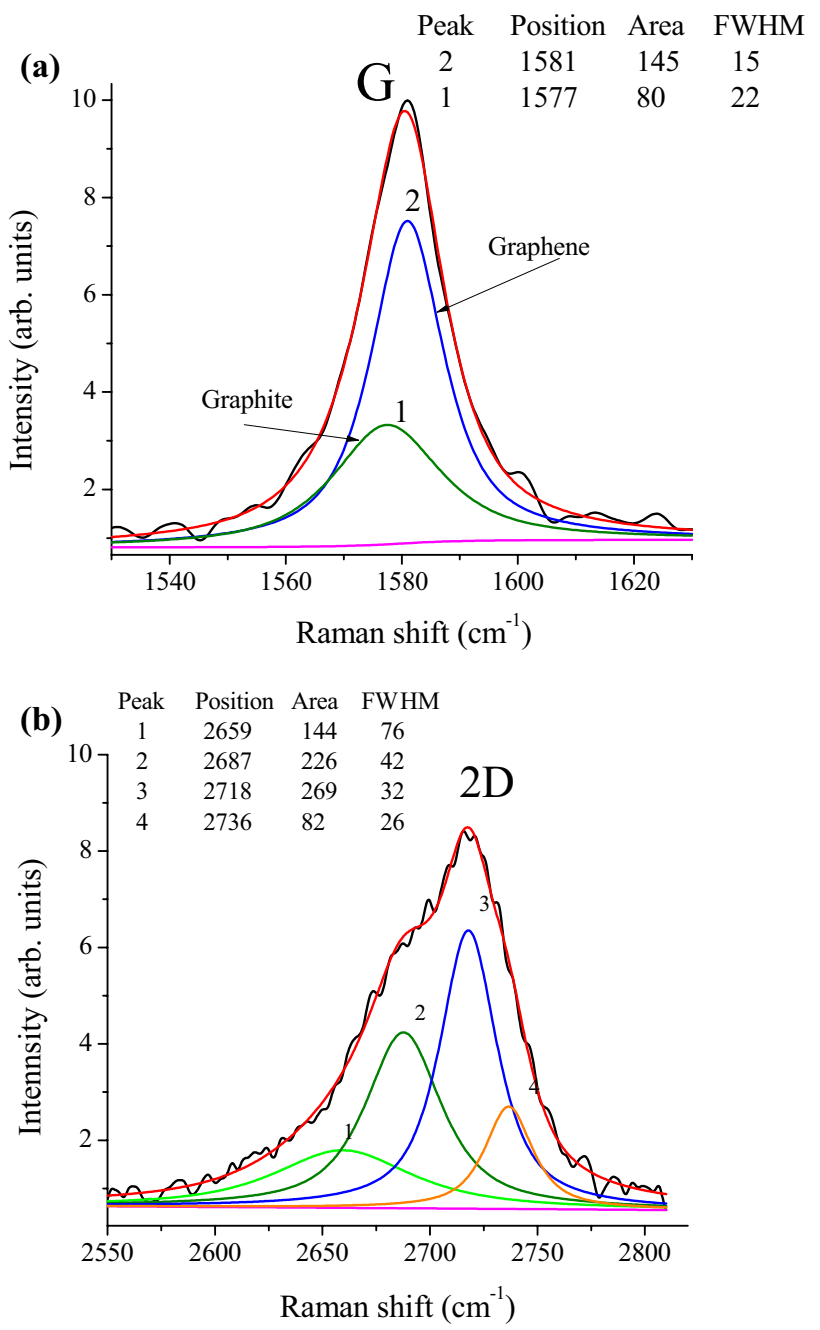

Fig. 4 Deconvolution of $\mathbf{a} G$ and $\mathbf{b}$ 2D Raman bands of the sample prepared at $0.5 \mathrm{~J} / \mathrm{cm}^{2}$ in an ultrasonic field

the $\mathrm{G}$ and 2D peaks were deconvoluted following the band adscription given in References [25, 26]. The results for samples produced at $0.5 \mathrm{~J} / \mathrm{cm}^{2}$ are shown in Fig. 4 .

Assuming that the $\mathrm{G}$ peak is formed by the convolution of two components, one due to graphite and another due to graphene, the deconvolution results reveal peaks at 1577 and $1581 \mathrm{~cm}^{-1}$, respectively. The peak assigned to graphene becomes approximately 1.8 times larger than the peak due to graphite. The $2 \mathrm{D}$ band has been employed to determine the graphene layer number because it strongly affects the band shape and is also clearly different in graphene and graphite [18]. Figure $4 \mathrm{~b}$ shows the results of the deconvolution of the 2D band. The shape and the parameters of the deconvolution, as insets in Fig. 4, are consistent with bilayer graphene in good agreement with previous reports [25].

The ratio between the intensity of $2 \mathrm{D}$ and $\mathrm{G}$ peaks, $2 \mathrm{D} / \mathrm{G}$ has been used as an indicative of the number of graphene layers present, a value around 2.0 is associated 
with a graphene monolayer, whereas lower values correspond to a few layers of graphene. Figure 5a, shows the Raman spectra of samples prepared at different laser fluences under ultrasonic excitation. Again, these spectra were scaled to have similar intensity of the $G$ peak. It is clearly seen that as the laser fluence increases the $2 \mathrm{D}$ band intensity decreases suggesting a greater number of graphene-stacked layers. These spectra were deconvoluted and the $2 \mathrm{D} / \mathrm{G}$ ratio was determined using the areas under the peaks labeled as 2 . Figure $5 b$, shows the $2 D / G$ ratio for the samples prepared with ultrasounds at different laser fluences. A decrease of the $2 \mathrm{D} / \mathrm{G}$ ratio as the laser fluence increases due to the increasing number of stacked graphene layers is observed, most likely due to the higher number of produced layers and the increased probability of interaction between them.
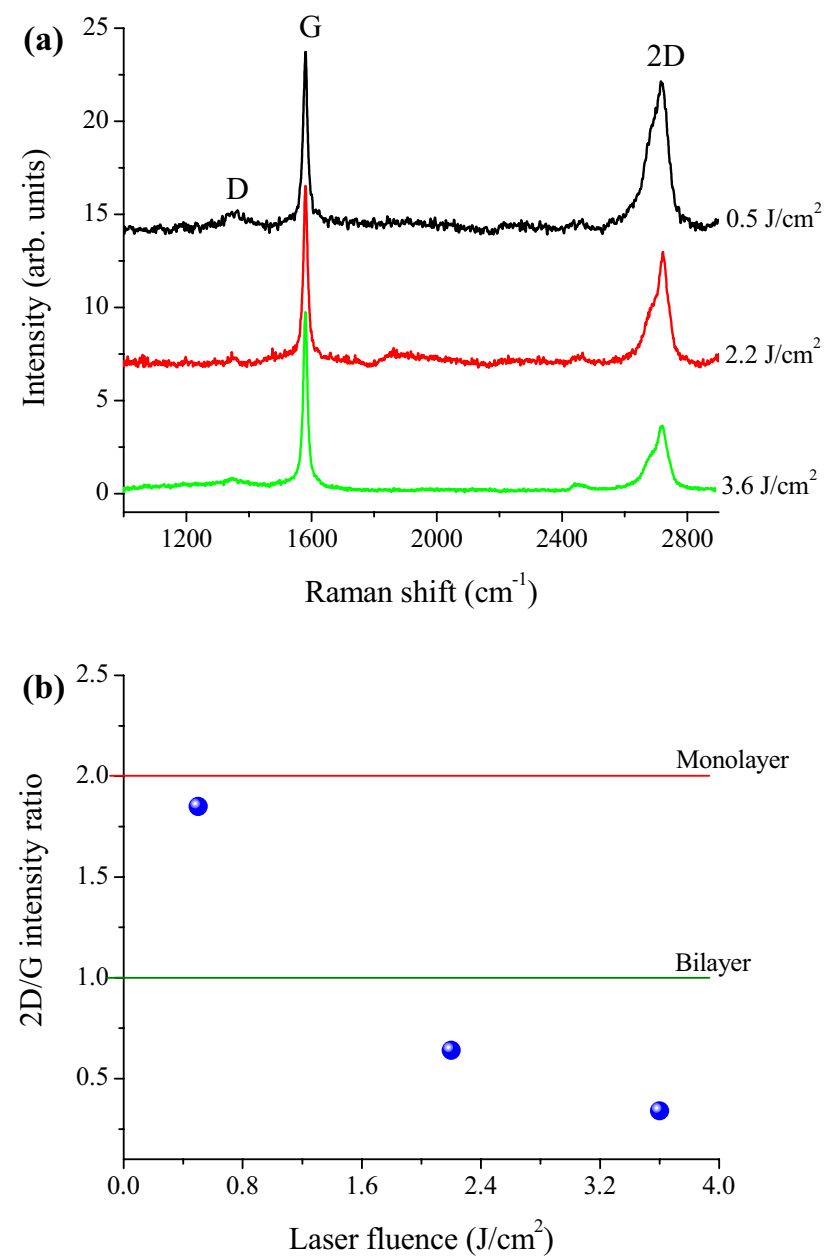

Fig. 5 a Raman spectra of samples prepared at different laser fluences under ultrasonic excitation. b Effect of laser fluence on the $2 \mathrm{D} / \mathrm{G}$ ratio for samples prepared at different laser fluences

\subsection{Proposed mechanism of nanosheet formation}

Laser ablation of graphite at $\lambda=532 \mathrm{~nm}$ in the ns-regime is a photothermal process [16] in which the increase of target temperature induced by the laser pulse leads to material melting or fracture and finally, to the ejection of ablated products from the target surface. In the case of graphite, this may favor the ejection of single or multiple graphene layers [16]. The presence of a liquid environment during ablation completely changes the expansion dynamics of the laser-ablated products due to the strong confinement of the produced plasma caused by the liquid media [14, 26]. A high-pressure shockwave forms and it propagates in both the liquid and in the target. This has been reported to induce exfoliation of graphite in the case of HOPG graphite targets [14]. In addition to this, a high-pressure, hightemperature cavitation bubble forms in the liquid when the plasma cools. This cavitation bubble, which contains a fraction of the ablated material, expands and shrinks and undoubtedly plays an important role on the nature and morphology of the ablation products [26]. However, in our present experimental conditions, TEM analysis shows that laser ablation in liquids by itself leads to a mixture on carbon aggregates and graphene layers, being necessary ultrasonic excitation to increase the fraction of graphene in the samples. On the other side, ultrasonic excitation alone does not lead to any carbon product in the liquid in the present experimental conditions, although it has been reported that the use of ultrasonic excitation alone allows producing graphene from HOPG graphite in liquids [18]. In this case, shear-forces and cavitation induce exfoliation of the graphite target. Cavitation creates shockwave damage on the target, which weakens Van Der Waals forces between adjacent graphene layers. This combined with shear forces favors graphene exfoliation. This difference in behavior may be related to the fact that the power of the ultrasonic excitation used in this work is not high enough to exfoliate the graphite target or to the use a non-HOPG graphite target as deduced form the Raman spectra shown in Fig. 3.

The best results are obtained in the present work when combining laser ablation and ultrasonic excitation. In this case, laser ablation generates carbon aggregates and most likely multiple-layer lamellar structures due to shockwave exfoliation, while ultrasonic vibrations in liquid media inside a container are transformed into stationary waves which are able to vibrate the present lamellar structures. The induced vibration enables overcoming the Van der Waals forces that keep bonded the lamellar units inducing their exfoliation to give rise to the formation of individual and few layer sheets [17]. 


\subsection{Optical properties}

We have finally characterized the optical properties of the produced colloids. Their UV-Vis spectra are presented in Fig. 6. Samples prepared using ultrasonic excitation have a greater absorbance than samples without ultrasound, with a reduction in transmittance of approximately three times due to the presence of a larger amount of material and suggesting a more efficient production of carbon nanostructures. Additionally, the absorbance is found to increase as the laser fluence increases consistent with a greater amount of material ablated at higher fluences. All spectra show an absorption band peaking close to $267 \mathrm{~nm}$ attributed to the $\pi-\pi^{*}$ transitions of aromatic $\mathrm{C}-\mathrm{C}$ bonds, in good agreement with previous reports for graphene $[27,28]$. The PL spectra corresponding to samples upon excitation at $320 \mathrm{~nm}$ with and without ultrasonic excitation are shown in Fig. 6b. A blue photoluminescent emission is observed around $421 \mathrm{~nm}$ for the sample without ultrasound, whereas a slight blue-shift is
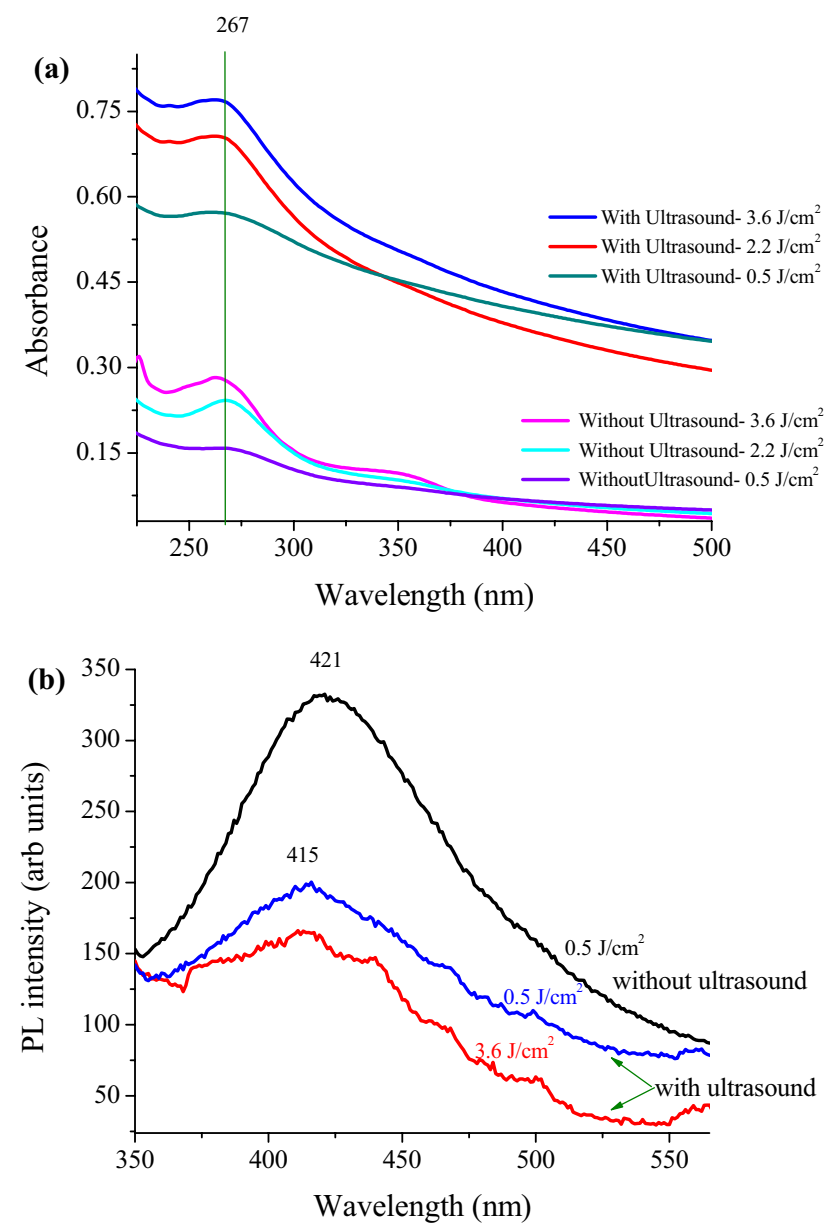

Fig. 6 a UV-Vis absorption spectra and b photoluminescent emission for the samples prepared with and without ultrasound of the obtained carbon colloids detected for samples with ultrasound, which would be consistent with a decrease of the size of the graphene quantum dots present in the sample produced with ultrasound [28]. Still, the overall emission intensity is apparently smaller in these samples in spite of showing a larger absorption. While the origin of this behavior is unclear at present, we may speculate with the presence of size and shape effects affecting both the position of the absorption peak and emission wavelength in a non-monotonic manner as shown in [28].

\section{Conclusions}

2D carbon nanostructures, specifically graphene with one to up to four layers, were successfully obtained combining liquid laser ablation with an ultrasonic field for the first time. Without ultrasound, carbon nanosheets agglomerated (stacked) combined with carbon nanoparticles were obtained. The presence of the ultrasonic field promotes the exfoliation of nanosheets producing graphene with one up to four layers as was confirmed by direct observation using transmission electron microscopy and Raman spectroscopy. The obtained results confirm that the combination of laser ablation in liquid media with an ultrasonic field can have potential advantages to produce $2 \mathrm{D}$ nanostructures.

Acknowledgements This work was partially supported by the CONACYT project CB-240998 and the Spanish project MINECO/FEDER TEC2015-69916-C2-1-R. Additionally, ININ through the project CB-602 is acknowledged. M. Martinez-Orts acknowledges the financial support of the Autonomous Community of Madrid and the European Social Fund (contract E-28-2017-0679526). The technical support with TEM measurements provided by P. Castillo of the electron microscopy laboratory of UAMI is also recognized.

\section{References}

1. X. Li, L. Tao, Z. Chen, H. Fang, X. Li, X. Wang, J.-B. Xu, H. Zhu, Appl. Phys. Rev 4, 021306 (2017)

2. Z. Liu, S.P. Lau, F. Yan, Chem. Soc. Rev 44, 5638 (2015)

3. C. Lee, H. Yan, L.E. Brus, T.F. Heinz, J. Hone, S. Ryu, ACS Nano 4, 2695 (2010)

4. L. Tang, R. Ji, X. Li, G. Bai, C.P. Liu, J. Hao, J. Lin, H. Jiang, K.S. Teng, Z. Yang, S.P. Lau, ACS Nano 8, 6312 (2014)

5. G. Bai, S. Yuan, Y. Zhao, Z. Yang, S.Y. Choi, Y. Chai, S.F. Yu, S.P. Lau, J. Hao, Adv. Mater 28, 7472 (2016)

6. R. Deng, Y. Zheng, G. Zhao, Cheng, ACS Nano 6, 3727 (2012)

7. M. V.Amendola, Meneghetti, Phys. Chem. Chem. Phys. 15, 3027 (2013)

8. S.I. Dolgaev, A.V. .Simakin, V.V. .Voronov, G.A. Shafeev, F. Bozon-Verduraz, Appl. Surf. Sci., 186, 546 (2002)

9. V. Amendola, S. Scaramuzza, F. Carraro, E. Cattaruzza, J. Colloid Interface Sci. 489, 18 (2017)

10. A. Singh, J. Vihinen, E. Frankberg, L. Hyvärinen, M. Honkanen and E. Levänen, Leo Hyvärinen, Mari Honkanen and Erkki Levänen. Nanoscale Res. Lett. 11, 447 (2016) 
11. A. Al-Hamaoy, E. Chikarakara, H. Jawad, K. Gupta, D. Kumar, M.S. R. Rao, S. Krishnamurthy, M. Morshed Appl. Surf. Sci. 302, 141 (2014)

12. D. Amans, M. Diouf, J. Lam, G. Ledoux, C. Dujardin, J. Colloid Interface Sci 489, 114 (2017)

13. E. Vaghri, D. Dorranian, Studia Ubb Chemia, LXI 4, 277 (2016)

14. M. Qiana, Y. S. Zhou, Y. Gao, T. Feng, Z. Sun, L. Jiang, Y. F. Lu. Appl. Surf. Sci 258, 9092 (2012)

15. Z. Yang, J. Hao, J. Mater. Chem. C 4, 8859 (2016)

16. M. Qian, Y. S. Zhou, Y. Gao, J. B. Park, T. Feng, S. M. Huang, Z. Sun, L. Jiang, Y. F. Lu. Appl. Phys. Lett. 98, 173108 (2011)

17. V. Šteng, J. Henych, M. Slušná, P. Ecorchard. Nanoscale Res. Lett. 9, 167 (2014)

18. K. Muthoosamy, S. Manickam, Ultrasonics-Sonochemistry 39, $478(2017)$

19. G. Lina, D. Tanc, F. Luoa, D. Chena, Q. Zhaoa, J. Qiud, Z. Xua, J. Alloys Compd. 507, L43 (2010)

20. N. Takada, A. Fujikawa, N. Koshizaki, K. Sasaki, Appl. Phys. A 110, 835 (2013)
21. S. Dadras, P. Jafarkhanu, M.J. Torkamany, J. Sabbaghzadeh, J. Phys. D Appl. Phys 42, 025405 (2009)

22. L. Escobar-Alarcón, E. Velarde Granados, D.A. Solís-Casados, O. Olea-Mejía, M. Espinosa-Pesqueira, E. Haro-Poniatowski, Appl. Phys. A 122, 433 (2016)

23. X.D. Ren, R. Lui, L.M. Zheng, Y.P. Ren, Z.Z. Hu, H. He,; Appl. Phys. Lett. 108, (Art. N. 071904) (2016)

24. C. Ferrari, J.C. Meyer, V. Scardaci, C. Casiraghi, M. Lazzeri, F. Mauri, S. Piscanec, D. Jiang, K.S. Novoselov, S. Roth, A.K. Geim, Phys. Rev. Lett. 97, 187401 (2006)

25. A. C. Ferrari, D. M. Basko, Nat. Nanotechnol. 8, 235 (2013)

26. C. Ferrari, Solid State Commun. 143, 47-57 (2007)

27. F.T. Johra, J.-W. Lee, W.-G. Jung, J. Ind. Eng. Chem. 20, 2883 (2014)

28. S. Kim, S. W. Hwang, M.-K. Kim, D. Y. Shin, D. H. Shin, C. O. Kim, S. B. Yang, J. H. Park, E. Hwang, S.-H. Choi, G. Ko, S. Sim, C. Sone, H. J. Choi, S. Bae, B. H. Hong. ACS Nano 6(9), 8203-8208 (2012) 